\title{
Gastric Cancer in Aktobe Region of Western Kazakhstan from 2009 to 2018: Incidence Rates, Trends, and Five-Year Survival
}

\author{
Saule K Balmagambetova ${ }^{1 *}$, Yerbol Z Bekmukhambetov ${ }^{1}$, Anar B \\ Tulyaeva', Yerbolat M Iztleuov', Gaziza A Smagulova', Arip K Koyshybaev', \\ Olzhas N Urazayev ${ }^{1}$, Saganaj T Djussembekov ${ }^{1}$, Valeriy V Begunov ${ }^{1}$, Irakli \\ Kokhreidze $^{2}$
}

\begin{abstract}
Objective: to assess the current state of gastric cancer (GC) incidence and its five-year survival across Aktobe region of western Kazakhstan from 2009 to 2018 by presenting key indicators and analyzing the most significant features. Methods: Rough incidence rates (per 100,000) and average annual percent changes (aAPCs) were estimated for each age group at diagnosis with respect to gender, ethnicity, residence, the disease stages, tumor subsite, and histology type using linear regression analysis, including the prognostic index for 2019-2020. Overall five-year survival rates were estimated by the Kaplan-Meier method. Results: Overall GC incidence increased from 19.2 to 29.3, and averaged 25.8 (R2 0.65 ) with aAPC of $3.2 \%$, with a potential to further rise ( 30.4 by $2020, \mathrm{p}<0.001)$. Non-cardia location (17.8, $\mathrm{p}<0.001$, aAPC $6.4 \%)$ and intestinal type of the tumor $(17.0, \mathrm{p}<0.001$, aAPC $7.35 \%)$ were predominant. The observed overall five-year survival rate was $28.4 \%(95 \%$ CI $24.5 ; 32.3)$ with a median survival time of 8.0 months $(95 \%$ CI $6.6 ; 9.4)$. Groups aged $40-49$ and $\geq 70$ had the lowest rates $(24.4 \%$ and $22.1 \%$, respectively, log-rank p 0.008$)$, but the youngest individuals (18-39 years) showed the shortest median survival time, 5.0 months after diagnosis at the survival rate of $29.4 \%$. Resectional surgery contributed significantly to the median survival time, 23.0 months vs. 6.0 in non-operated patients (log-rank $\mathrm{p}<0.001)$. Conclusion: $\mathrm{GC}$ in Aktobe region was featured by growing incidence and unsatisfactory five-year survival rates. Indigenous males of 60-69 years old with intestinal histology type, as well as the youngest patients irrespective of their gender, ethnicity, and other characteristics were recognized as high risk groups. Besides, relatively high aAPC 5.1\% in the youngest revealed their further expected vulnerability. Further research is suggested to focus on risk factors, including gene expression profiling, to find out an accessible preventive strategy.
\end{abstract}

Keywords: Gastric cancer- incidence- trends- five-year survival- western Kazakhstan

Asian Pac J Cancer Prev, 21 (6), 1645-1652

\section{Introduction}

According to the International Agency for Research on Cancer (IARC), Gastric cancer (GC) remains one of the most deadly neoplasms worldwide, particularly among older males. It is the fifth most common malignancy and the third leading cause of death in both sexes after lung cancer and colorectal cancer (Bray et al., 2018). Since gastric cancer has a multifactorial nature and it is one of the most behaviorally influenced cancers, its incidence is highly variable by region and culture (Rawla and Barsouk, 2019). In Eastern and Central Asia and Latin America, the highest incidence rates of $\mathrm{GC}$ are recorded (Balakrishnan et al., 2017). Researchers from Brazil also pointed out the highest GC incidence in Belem, 69.1/100,000 in men (2003) and 26.7/100,000 in women (2006) (Curado et al., 2019). The Republic of Korea has reported the highest national incidence of GC with almost 60 per 100,000 new cases annually for males (Ferlay et al., 2018). On the contrary, its overall incidence in the United States has been steadily declined over the past 75 years. The National Cancer Institute's Surveillance, Epidemiology, and End Results (SEER)estimated that about 1/114 men and women will be diagnosed with GC during their life in 2009 (Lee et al., 2013). Meanwhile, this highly lethal tumor still is featured by relatively low five-year survival, within or less than $30 \%$ on a global scale (Crew and Neugut, 2006).

The most predominant non-cardia cancers are brought about by chronic gastritis caused by Helicobacter pilori or other inflammation of the stomach lining which can be caused by a variety of environmental factors (Mukaisho et al., 2015). Along with this, it has been proven that the familial clustering of $\mathrm{GC}$ is seen in $10 \%$ of cases, and 
approximately $3 \%$ of cases arise in the setting of hereditary diffuse GC (HDGC). All reported HDGCs are pure diffuse type according to Lauren classification (Lauren, 1965) and are associated with dismal prognosis once the tumor invades the submucosa (Luo et al., 2018). In families with HDGC, GC is presented at a relatively young age before 40 years old. Germline mutations in the $\mathrm{CDH} 1$ gene are the major cause of HDGC and are identified in approximately $25-50 \%$ of families that fulfill strict $\mathrm{CDH} 1$ testing criteria (van der Post et al., 2015). Accordingly, countries with a high incidence of sporadic gastric cancer, such as Japan and Korea, have a lower frequency of germline mutations in familial GCs than low-incidence countries. The cause of familial clustering in high-incidence countries is more likely environmental than hereditary (Vogelaar et al., 2012).

Within 2004-2013, the GC incidence rates in Kazakhstan fluctuated in frames of 21.8-25.6 per 100,000, with the peak rates in the age group of $\geq 70$ and a proportion of $8.5 \%$ in the general structure of malignancies. Besides, a tendency in morbidity reduction was recorded between 2004 and $2013(-18.0 \%)$, and the western region was referred to as the medium level zone of GC incidence (Orazova et al., 2015).

Among the risk factors for GC development, environmental pollution is not being accented comparing to the role of Helicobacter pilori, while the Aktobe province of western Kazakhstan, as well as the entire western region, is known by its ecology troubles due to presence of oil, chromium producing, and other industries. Environmental pollution is also related to the fact that the Aktobe province is partially located in the vast Aral Sea ecological disaster region. According to data, long-time average annual levels of cancer morbidity for 2004-2013 in adult population living in the Aral Sea environs were up to 2.6 times higher, compared to ecologically intact terrains. In particular, long-time average annual level of cancer morbidity in adult population living in that zone was $57.2 \%$ higher, and the total cancer morbidity depended on the hazard coefficient (HQ) associated mostly with the inhalation of nickel and the combined cadmium intake $(\mathrm{r}=0.8)$ (Mamyrbayev et al., 2016). Overall, the Aktobe province is highly indicative of cancer morbidity due to the mentioned reasons.

However, the analysis GC incidence rates and the disease's main features, such as the distribution of histology types, proportion of tumor subsites and stages at diagnosis, dynamics across the age groups, etc., has not been performed by local researchers yet.

Thus, the present study aimed to assess the current state of GC incidence and its five-year survival across Aktobe province of the western region by presenting key indicators and analyzing the most significant features.

\section{Materials and Methods}

\section{Study setting}

This retrospective database research was approved by the University's IREC (Protocol No. 24, 24.05.2019) and performed following the Helsinki Declaration principles. Informed consent was not required as the individually identifiable data of patients were not involved.

The present research scopes terrain of the Aktobe province, the largest in western Kazakhstan (Central Asia). Indigenous inhabitants are Kazakhs constituting $82.4 \%$ of the total population, of Turkic ethnic group, followed by Slavs and other ethnic groups (Azerbaijanians, Koreans, Tatars, Germans, etc.). Population density in western region is the smallest in the country, 3.4 per $\mathrm{km}^{2}$, according to data of the National Statistics Agency in 2016.

\section{Study population}

Data on the province's total adult population $(\geq 18)$ from 2009 to2018 (522,282 in January 2009 and 587,544 in January 2018), including males/females, and by age groups were requested from the Aktobe Statistical Committee.

All incident first diagnosed GC cases (C16.0-C.16.9, The WHO ICD-10 Version: 2016) from 2009 to 2018 in adults aged 18 years and older were obtained from the Cancer Registry of the Aktobe Regional Oncologic Center. Rough incidence rates (per 100,000) and average annual percent changes (aAPCs), as well as the ratios were estimated for each age group at diagnosis (18-39, 40-49, $50-59,60-69$, and $\geq 70$ years), with respect to gender, ethnicities (Kazakhs and others), residence (urban or rural area), and the disease clinical stage (The $8^{\text {th }}$ edition of the UICC TNM classification, 2016: gastric carcinoma, adenocarcinoma). Stages were presented as St I; St II (St IIA + St IIB $\left.-\mathrm{T}_{1-4 \mathrm{a}} ; \mathrm{N}_{0-3}, \mathrm{M}_{0}\right)$; St III; and St IV $\left(\mathrm{T}_{4 \mathrm{~b}}\right.$, any $\mathrm{T}$; any $\left.\mathrm{N} ; \mathrm{M}_{0-1}\right)$.

Regarding the histology type, we matched definitions presented by Lauren's and WHO classifications: "indeterminate" type by Lauren is defined as a mixed type by the WHO. "Adenocarcinoma with mixed subtypes, or Adenocarcinoma combined with other types of carcinoma" is encoded as $8255 / 3$ in the WHO third edition of the International Classification of Diseases for Oncology (ICD-O), 2013 (Berlth et al., 2014). Some authors missed this histologic type referring to as the previous classifications, where the mixed type was the part of diffuse type, but in our Registry, the mixed type was recorded in the amount of $9.6 \%$. Overall, all GC cases in the present paper were assigned into three histologic types: diffuse, intestinal, and mixed ones.

Categorization of cases by the sites was depended on the number of cases. Keeping in mind the fact that the number of C16.8 and C16.9 cases in our Registry was negligibly small (15 out of 1,458 ), we merged non-cardia sites (C16.1-C16.6) with codes 16.8-16.9. A tumor was coded 16.8 (overlapping lesion of the stomach), when the location of the lesion was in the anterior or posterior wall of the stomach and none of the specific sites was mentioned, and cases with code 16.9 (stomach, NOS) were cases with missing site codes. So, this research dealt with two main sublocations, namely C16.0 - cardia, C16.1-C16.9 - non-cardia, and other GC cases. The observed five-year survival rates were also stratified by age group, histology type, clinical stage, the tumor subsite, as well as resectional surgery. 


\section{Data analysis}

Calculations were done in Statistica.10 (Dell Technologies, Round Rock, Texas, USA) as well as SPSS (version 25) (IBM, Armonk, USA). For all tests, a two-sided type I error of $\mathrm{p}<0.05$ at $95 \%$ confidence interval (CI) was assumed statistically significant. Rough incidence rates and corresponding 95\% CIs were determined by linear regression analysis and expressed as the number of cases per 100,000 individuals, including the prognostic index for 2019-2020. Changes in the incidence rates were presented as a summary measure of a trend over a fixed period of time and expressed as aAPCs. Five-year survival rates were estimated through commonly accepted Kaplan-Meier analysis by defining the differences between groups through the log-rank criterion.

\section{Results}

In the Aktobe Cancer Registry, a total of 1,458 records of patients who were first diagnosed with GC over the
2009-2018 period were found. Descriptive statistics of GC patients is presented in the first two columns of Table 1.

Kazakh men aged $\geq 60$ with non-cardia location of tumor and predominance of intestinal histology type had the highest prevalence. Besides, most of them were at stage II or III at the time of diagnosis; whereas, cases registered with stage I had the lowest prevalence (4\%). The residence of patients with GC did not have a significant effect on.

Overall dynamics in incidence by year evidenced that an increase in GC rate from 19.2 in 2009 up to 29.3 in 2018 was featured by uneven spasmodic growth with a relative outbreak of unexplained nature in 2010 and a smooth increase starting since 2015. Such a pattern was more expressive in men compared to women. The proportion of stages I and II (resectable) was not increased for a decade as noticeably as advanced stages of III and IV ( 9.3 to 13.3 vs. 9.2 to 16.0 , respectively). Non-cardia location prevalence increased almost twice (from 12.8 up to 22.3), unlike the cardia site of the tumor. Regarding the histology types, the prevalence of intestinal type also

Table 1. Descriptive Characteristics of Population First Diagnosed with GC and the Rough Incidence Rates for All Studied Parameters and Population Groups, Including Trends

\begin{tabular}{|c|c|c|c|c|c|c|c|c|c|}
\hline Population groups & $\mathrm{N}$ & $\%$ & $\begin{array}{c}\text { Rate } \\
\text { (un-adjusted) }\end{array}$ & St. error & $95 \%$ CI, lower & $\begin{array}{c}95 \% \\
\text { CI, upper }\end{array}$ & Ratio & $P$-value & aAPC, $\%$ \\
\hline Total & 1,458 & 100 & 25.8 & 2.1 & 21.6 & 30 & & & 3.2 \\
\hline \multicolumn{10}{|l|}{ Gender: } \\
\hline Females & 490 & 33.6 & 8.7 & 1.2 & 6.3 & 11.1 & ref. & & 3.1 \\
\hline Males & 968 & 66.4 & 17.1 & 1.7 & 13.7 & 20.5 & 2 & $<0.001$ & 3.2 \\
\hline \multicolumn{10}{|l|}{ Ethnicity: } \\
\hline Others & 299 & 20.5 & 5.7 & 0.4 & 4.9 & 6.5 & ref. & & -3.2 \\
\hline Kazakhs & 1,159 & 79.5 & 20.1 & 1.1 & 17.5 & 22.7 & 3.9 & $<0.05$ & 6.2 \\
\hline \multicolumn{10}{|l|}{ Residence: } \\
\hline Rural area & 691 & 47.4 & 12.6 & 0.5 & 11.4 & 13.8 & ref. & & 2.5 \\
\hline Urban area & 767 & 52.6 & 13.2 & 0.6 & 11.8 & 14.7 & 1.1 & 0.39 & 4 \\
\hline \multicolumn{10}{|l|}{ Age } \\
\hline $18-39$ & 60 & 4.1 & 0.9 & 0.1 & 0.7 & 1.3 & ref. & & 5.1 \\
\hline $40-49$ & 133 & 9.1 & 2.2 & 0.2 & 1.7 & 2.7 & 2.2 & $<0.001$ & 1.5 \\
\hline $50-59$ & 386 & 26.5 & 6.9 & 0.3 & 6.2 & 7.5 & 6.4 & $<0.001$ & 2.5 \\
\hline $60-69$ & 440 & 30.2 & 7.8 & 0.5 & 6.8 & 8.9 & 7.3 & $<0.001$ & 5.7 \\
\hline $70+$ & 439 & 30.1 & 7.9 & 0.4 & 7 & 8.8 & 7.3 & $<0.001$ & 2.3 \\
\hline \multicolumn{10}{|l|}{ Stage: } \\
\hline I & 58 & 4 & 0.9 & 0.2 & 0.6 & 1.4 & ref. & & 0.5 \\
\hline II & 706 & 48.4 & 12.3 & 0.8 & 10.5 & 14.1 & 12.2 & $<0.001$ & 4.5 \\
\hline III & 491 & 33.7 & 8.7 & 0.6 & 7.3 & 10.1 & 8.5 & $<0.001$ & 1.8 \\
\hline IV & 203 & 14 & 3.8 & 0.3 & 3.2 & 4.4 & 3.5 & $<0.001$ & 3.8 \\
\hline \multicolumn{10}{|l|}{ Tumor site: } \\
\hline C16.0 (cardia) & 480 & 32.9 & 7.9 & 0.5 & 6.8 & 9.1 & ref. & & -1.4 \\
\hline $\begin{array}{l}\text { other GC sites } \\
\text { (C16.1-C.16.9) }\end{array}$ & 978 & 67.1 & 17.8 & 0.9 & 15.5 & 19.9 & 2 & $<0.001$ & 6.4 \\
\hline \multicolumn{10}{|l|}{ Histology type: } \\
\hline Mixed & 140 & 9.6 & 2.6 & 0.3 & 1.8 & 3.3 & ref. & & 4.3 \\
\hline Diffuse & 402 & 27.6 & 6.2 & 0.8 & 4.3 & 8.1 & 2.9 & 0.061 & 3.5 \\
\hline Intestinal & 916 & 62.8 & 17 & 1.3 & 14.1 & 20 & 6.5 & $<0.001$ & 7.4 \\
\hline
\end{tabular}




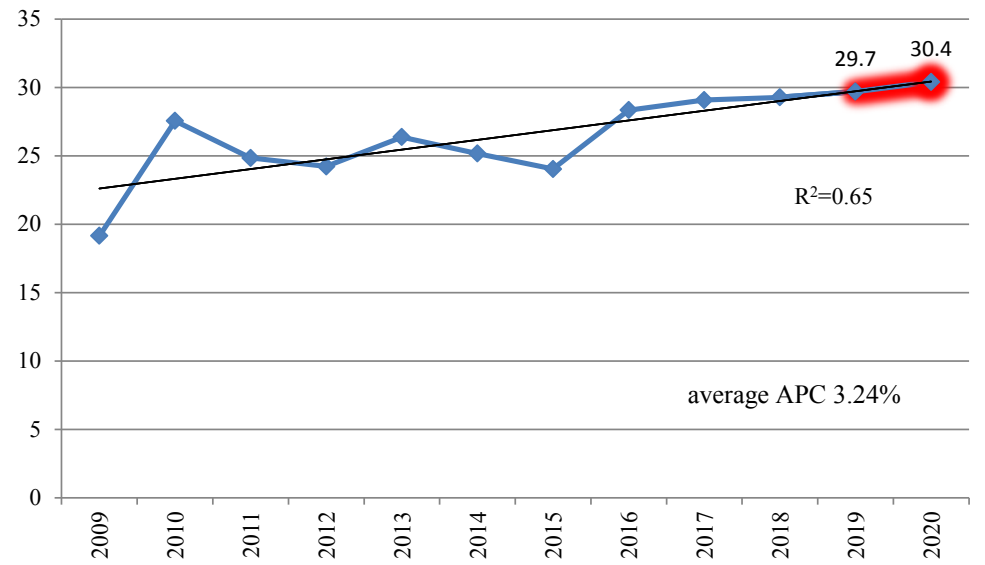

Figure 1. GC Incidence Over Years, Including Prognostic Indices for 2019-2020

incremented from 11.9 to 19.9 over the period compared to diffuse and mixed ones. The most active dynamics was observed in age groups of 40-49 and 60-69 years old. Overall incidence rate dynamics is reflected in Figure 1, where prognostic indices for 2019-2020 are also presented.

Thus, further increase in GC incidence rate from 19.2 in 2009 up to 29.7 (95\% CI $23.9 ; 32.7)$ in 2019 and to $30.4(95 \%$ CI $26.9 ; 33.5)$ in 2020 is expected $\left(\mathrm{R}^{2} 0.65\right.$, $\mathrm{p}<0.001)$. It is noteworthy to mention that this outbreak of 2010 was observed in the graph on incidence dynamics among Kazakh patients only, unlike other ethnicities.
Dynamics in incidence rates across various ethnicities is presented in Figure 2.

Table 1 also reflects overall data on the incidence by explored parameters and average aAPCs for different population groups.

Quite remarkable is an expected decrease in morbidity among the other ethnicities, unlike the Kazakhs, where the trend stayed positive (aAPC -3.2 vs. $6.2 \%$ ). Another negative trend, albeit non-significant, was noted in the cardia subsite of the tumor (aAPC $-1.4 \%$ ), where the proportion was $32.9 \%$ through the last decade. Regarding the age group, increasing trend in GC cases among

Table 2. Overall Five-Year Survival Rates with Respect to Age Group, Tumor Location, Histology Type, Clinical Stage, and Resectional Surgery $(\mathrm{N}=762)$.

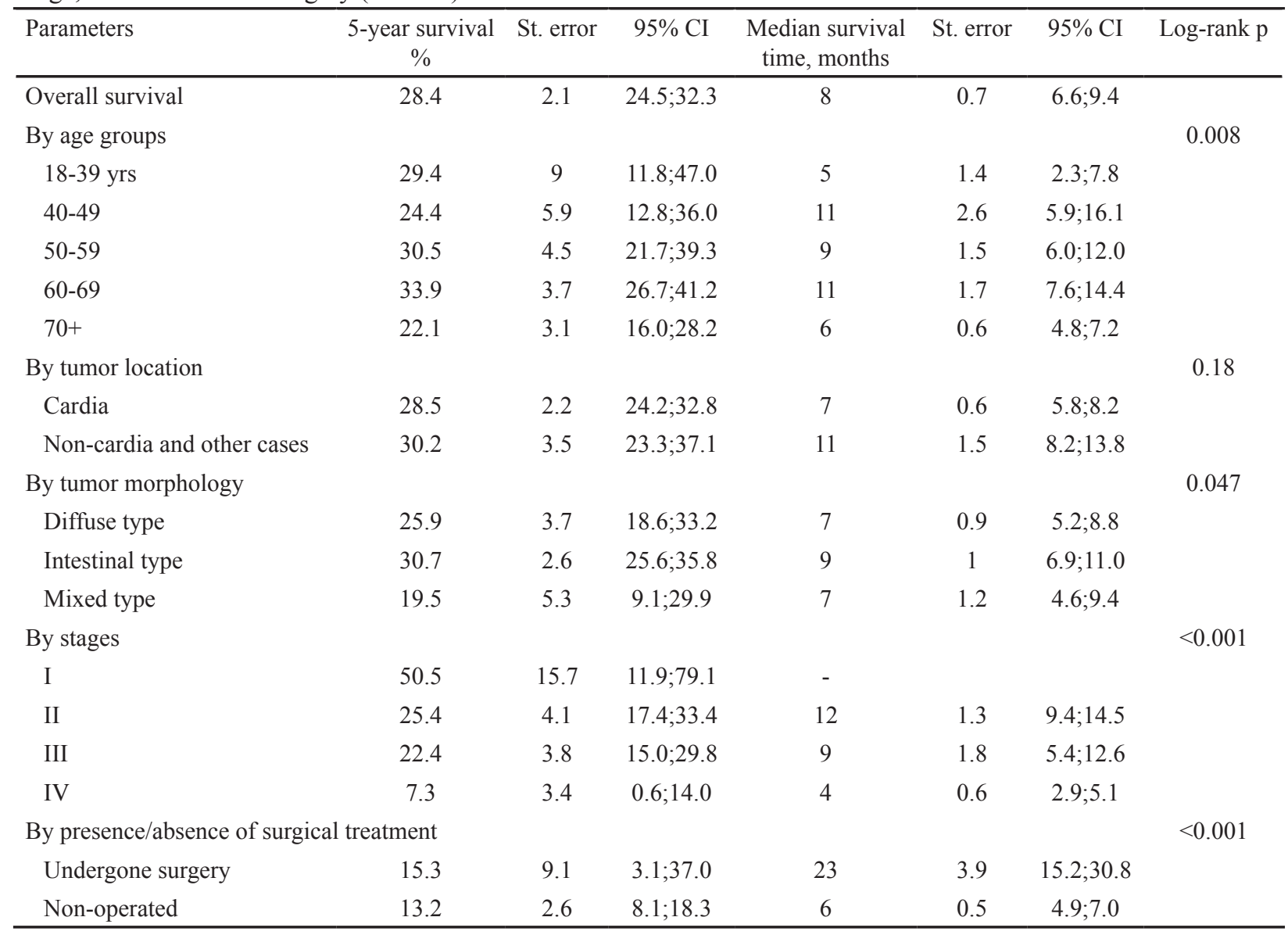




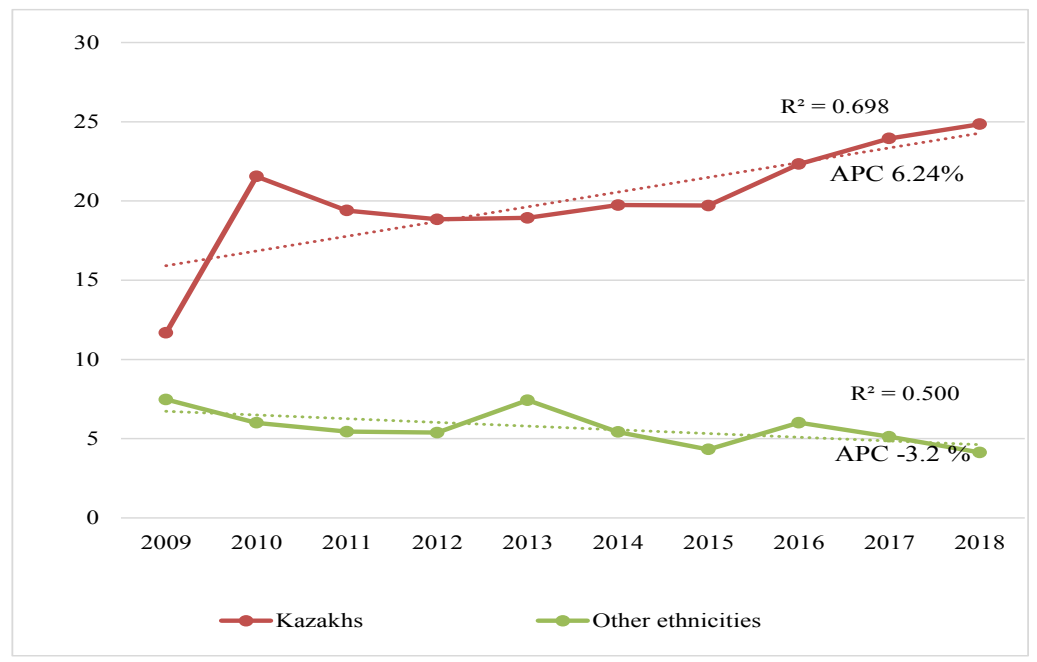

Figure 2. GC Incidence Across Various Ethnicities

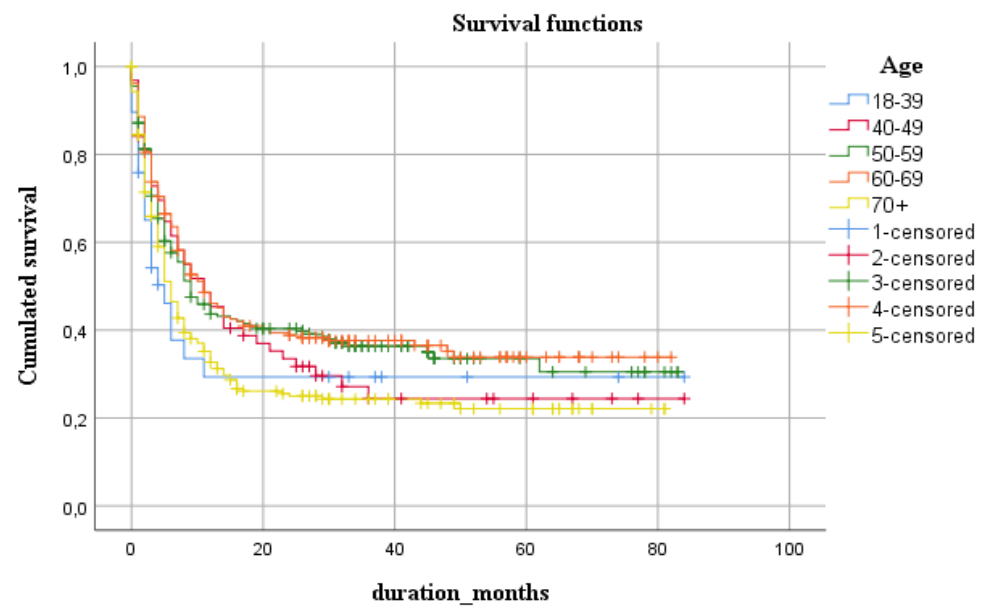

Figure 3. Five-Year Survival Across Different Age Groups

patients aged 60-69 and relatively sharp growth in its incidence (aAPC 5.1\%) among patients aged $<39$ years old was expected. Generally, young individuals are usually being diagnosed hereditary diffuse GC, but the trend in diffuse GC was relatively small compared to intestinal one (aAPC 3.5 vs. 7.4\%).

The observed five-year survival was calculated $(\mathrm{N}=762$ from 2014 to 2018 ) and stratified by age, tumor location, clinical stage, histology type, and resectional surgery. Overall five-year survival rate was calculated as $28.4 \%$ at the median survival time of 8 months. Obtained survival rates are summarized in Table 2 and in corresponding graphs.

Survival rate depending on clinical stage consecutively declined from $50.5 \%$ at stage I to $7.3 \%$.

Though the incidence rate smallest values were

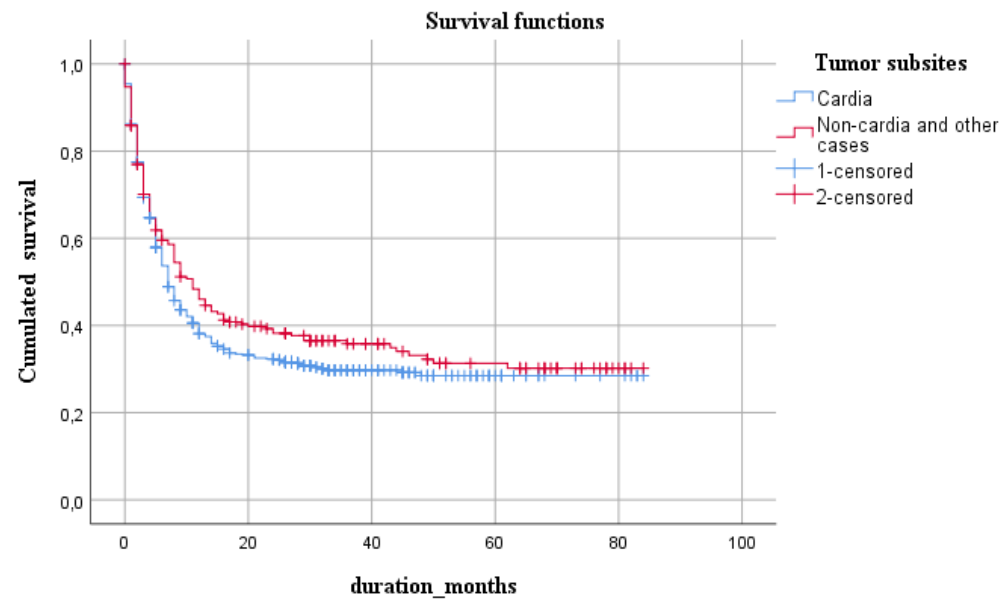

Figure 4. Five-Year Survival with Respect to Tumor Subsites 


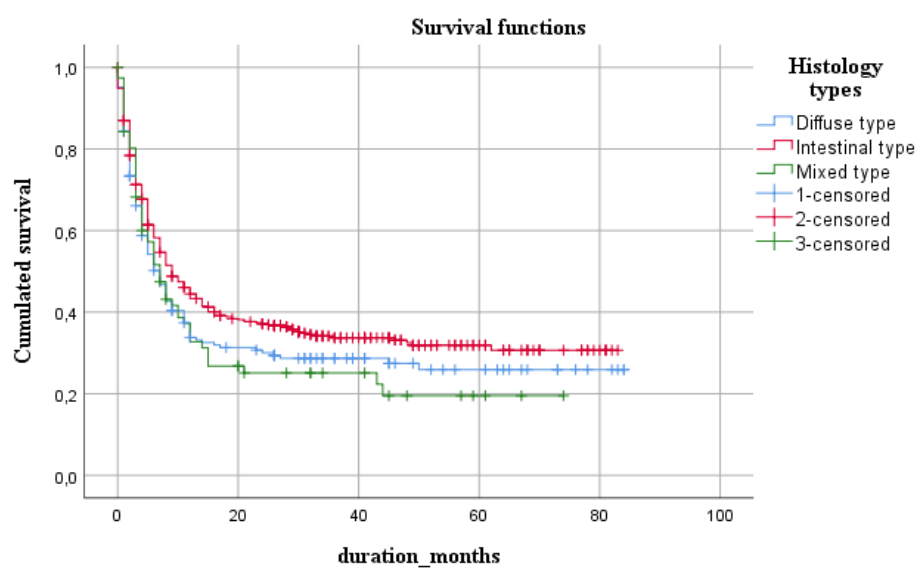

Figure 5. Five-Year survival with Respect to Tumor Morphology

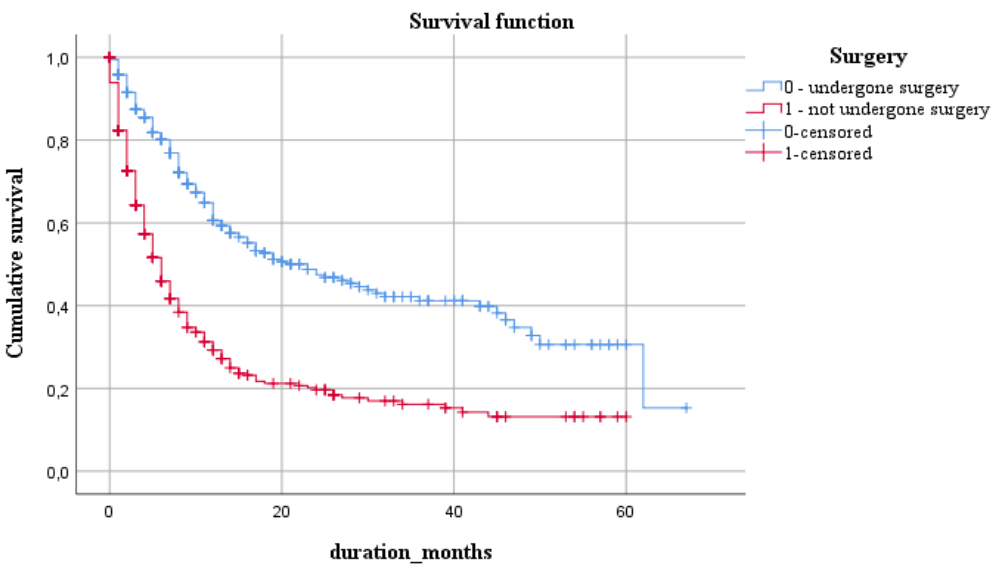

Figure 6. Five-Year Survival in Terms of Presence or Absence of Surgical Treatment

observed in the age groups of $40-49$ and $\leq 70$ years old, shorter median survival time was revealed in the youngest patients (5 months after diagnosis vs. 11 and 6 months, respectively).

Non-cardia location was the more favorable for survival time than the cardia site of the tumor ( 11 months vs. 7 months).

Patients with the intestinal type of tumor showed a relatively large rate, $30.7 \%$, as well as the survival time, 9 months, compared to other types.

Surgical treatment significantly impacted the median survival time, being 23 months in those who underwent surgery vs. 6 months in non-operated patients.

\section{Discussion}

Relatively quick growth in the GC incidence rates from 19.2 up to 29.3 in 2018 observed in the Aktobe region is similar to data obtained in the borderline Uralsk region for almost the same period reporting 22.5 to 27.5 in men and 8.5 to 16.0 in women (Umarova et al., 2016). Given that the incidence is incremented mostly among the indigenous population, this phenomenon calls for some reasonable explanation. Overall, there are globally evidenced racial, ethnic, and gender disparities in the GC incidence (Nagini, 2015; Luo et al., 2017). For instance, between 1988 and 2012 in California, Korean Americans had about five times greater incidence of GC than Non-Hispanic Whites and twice that of Japanese Americans (Lee et al., 2017). Similar disparities were also observed in our analysis detecting that men/women ratio was 2:1 and Kazakhs/ other ethnic groups ratio was 5:1. One of the reasons for ethnic disparities in GC might have been due to relatively fast transition into western nutrition style with a wide consuming of ultra-processed food; whereas, the traditional diet of Kazakhstan was of nomadic nature and mostly consisted of boiled meat and dairy. Meanwhile, a large number of research has proved that even a $10 \%$ increase in the proportion of ultra-processed food in the diet is associated with an increase of more than $10 \%$ in the risks of overall cancer, not counting other various consequences, such as metabolic syndrome or diabetes mellitus (Fiolet et al., 2018; GBD Diet Collaborators, 2019; Hall et al., 2019).

Obvious disparities between GC patients from East and West were described by Griniatsos and Trafalis in 2018. According to them, GCs in the East are mainly diagnosed at younger ages, they are of intestinal type, and diagnosed at an early stage of the disease, while GCs in the West are mainly affecting elderly patients, they are of diffuse type, and diagnosed at an advanced stage. In our analysis, we reported prevalence of the age group of 60$69(30.2 \%)$, intestinal histotype (62.8\%), and non-cardia location of the tumor $(67.1 \%)$, but we could not present the proportion of histology types and the tumor subsites by ethnic groups and their distribution by age. The mentioned 
researchers (Griniatsos and Trafalis) analyzed data from the US NCI's SEER registries (N 13,840) and reported the median overall survival of 6 months in patients aged $\leq 44$ years old compared to 3 months in patients aged 75 years old and older. Similarly, our analysis revealed quite similar data on median survival, revealing median survival of 5 months in those aged 18-39 years old and 6 months in patients aged $\geq 70$.

Based on the US SEER data, another study confirmed the racial disparities in the GC key indices in Asian and Caucasian patients. Although it reported significantly high median survival time among patients with IB, IIA, or IIB disease, the Asian patients had 37, 72, and 13 months longer median survival time than the corresponding Caucasian patients (Wang et al., 2015).

We obtained relatively similar survival rates in those underwent surgery and those who did not undergo surgery. $15.3 \%$ and $13.2 \%$, with corresponding survival time 23 months and 6, respectively. Researchers from Sweden reported that the relative postoperative 5-year survival increased up to $43-44 \%$; whereas, it stayed within 2-5\% in non-operated patients for the last two decades (Asplund et al., 2018).

In general, the five-year survival rate is relatively good only in Japan, where it reaches $90 \%$. In European countries, survival rate varies from $\sim 10 \%$ to $30 \%$. High survival rate in Japan is probably achieved by early diagnosis using endoscopic examinations and consecutive early tumor resection (Sitarz et al., 2018).

To our knowledge, there are a few prevention strategies to minimize GC lethality. Helicobacter pylori eradication therapy significantly reduced GC incidence by $39 \%$, as it was shown in the extended Shandong trial that lasted 14.3 years. Consensus groups from Asia, Europe, and Japan have recommended $H$. pylori eradication as primary prevention in high-risk areas. Following eradication therapy, endoscopic surveillance of pre-malignant lesions using enhanced imaging appears to be another effective preventive strategy (Fock, 2014). Pre-endoscopic risk assessment should be based on demographic and clinical features, such as ethnicity, age, gender, smoking, and H. pylori status. Nevertheless, just a few Asian countries with a high risk of GC have been applied population-based screening programs for daily practice (Quach et al., 2019). Another prospective way to prevent growing incidence GCof is the implementation of genetic counseling in relatives of patients with revealed of GC symptoms at an early age and suspected for hereditary diffuse GC (Moslim et al., 2018).

Overall, GC in the Aktobe region currently is featured by growing incidence and unsatisfactory five-year survival rate. Indigenous males aged 60-69 years old with intestinal histology type and the youngest patients irrespective their gender, ethnicity, and other characteristics are high risk groups. Besides, relatively high aAPC of $5.1 \%$ in the youngest reveals their further expected vulnerability.

Though the study introduced the current pattern of GC in western Kazakhstan, it had a number of limitations. One of the limitations was related to comparative shortness in analyzing the survival rate. Besides, it would be logically justified to present also the data on mortality through the region.

Low rates of the five-year survival, particularly among the youngest, call for an in-depth analysis of the treatment and prevention tactics practiced by health providers in the region.

Further research is suggested to focus on risk factors, including gene expression profiling, to find out an accessible preventive strategy.

\section{Acknowledgments}

\section{Funding statement}

The research was funded by the Marat Ospanov West Kazakhstan Medical University.

\section{Conflict of interest}

The authors declare that they have no conflict of interest.

\section{References}

Asplund J, Kauppila JH, Mattsson F, Lagergren J (2018). Survival trends in gastric adenocarcinoma: A populationbased study in Sweden. Ann Surg Oncol, 25, 2693-702.

Balakrishnan M, George R, Sharma A, Graham DY (2017). Changing trends in stomach cancer throughout the world. Curr Gastroenterol Rep, 19, 36.

Berlth F, Bollschweiler E, Drebber U, Hoelscher AH, Moenig S (2014). Pathohistological classification systems in gastric cancer: Diagnostic relevance and prognostic value. World $J$ Gastroenterol, 20, 5679-84.

Bray F, Ferlay J, Soerjomataram I, Siegel RL, et al (2018). Global cancer statistics 2018: GLOBOCAN estimates of incidence and mortality worldwide for 36 cancers in 185 countries. $C A$ Cancer J Clin, 68, 394-424.

Crew CD, Neugut AI (2006). Epidemiology of gastric cancer. World J Gastroenterol, 12, 354-62.

Curado MP, Mendonca e Silva DR, Moura de Oliveira M, Soares F, Dirlei Begnami M (2019). Disparities in epidemiological profile of gastric adenocarcinoma in selected cities of Brazil. Asian Pac J Cancer Prev, 20, 2253-8.

Ferlay J, Ervik M, Lam F, et al (2018). Global cancer observatory: Cancer Today. Lyon, France: International Agency for Research on Cancer (Accessed 09 October, 2018). https://gco.iarc.fr/today.

Fiolet T, Srour B, Sellem L, oet al (2018). Consumption of ultra-processed foods and cancer risk: results from Nutri Net-Santé prospective cohort. BMJ, 360, k322.

Fock KM (2014). Review article: the epidemiology and prevention of gastric cancer. Aliment Pharmacol Ther, 40, 250-60.

GBD Diet Collaborators (2019). Health effects of dietary Risks in 195 countries, 1990-2017: a systematic analysis for the global burden of disease study 2017. Lancet, 393, 1958-72.

Griniatsos J, Trafalis D (2018). Differences in gastric cancer surgery outcome between East and West: differences in surgery or different diseases?. J BUON, 23, 1210-5.

Hall KD, Ayuketah A, Brychta R, et al (2019). Ultra-processed diets cause excess calorie intake and weight gain: An Inpatient Randomized Controlled Trial of Ad Libitum Food Intake. Cell Metab, 30, 67-77.

Lauren P (1965). The two histological main types of gastric carcinoma: diffuse and so-called intestinal-type carcinoma. An attempt at a histo-clinical classification. Acta Pathol Microbiol Scand, 64, 31-49.

Asian Pacific Journal of Cancer Prevention, Vol $21 \quad 1651$ 
Lee E, Liu L, Zhang J, Stern MC, Barzi A (2017). Stomach cancer disparity among Korean Americans by tumor characteristics: Comparison with Non-Hispanic Whites, Japanese Americans, South Koreans, and Japanese. Cancer Epidemiol Biomarkers Prev, 26, 587-96.

Lee S-M, Kim K-M, Ro JY (2013). Gastric carcinoma: Morphologic classifications and molecular changes. In: "Gastric Carcinoma - New Insights into Current Management”, edited by Daniela Lazar, Intech Open. DOI: $10.5772 / 54617$.

Luo G, Zhang Y, Guo P, et al (2017). Global patterns and trends in stomach cancer incidence: Age, period and birth cohort analysis. Int $J$ Cancer, 141, 1333-44.

Luo W, Fedda F, Lynch P, Tan D (2018). CDH1 gene and hereditary diffuse gastric cancer syndrome: Molecular and Histological Alterations and Implications for Diagnosis And Treatment. Front Pharmacol, 9, 1421.

Mamyrbayev A, Djarkenov T, Dosbayev A, et al (2016). The incidence of malignant tumors in environmentally disadvantaged regions of Kazakhstan. Asian Pac J Cancer Prev, 17, 5203-9.

Moslim MA, Heald B, Tu C, et al (2018). Early genetic counseling and detection of $\mathrm{CDH} 1$ mutation in asymptomatic carriers improves survival in hereditary diffuse gastric cancer. Surgery, 164, 754-9.

Mukaisho K, Nakayama T, Hagiwara T, et al (2015). Two distinct etiologies of gastric cardia adenocarcinoma: interactions among pH, Helicobacter pylori, and bile acids. Front Microbiol, 6, 412.

Nagini S (2015). Carcinoma of the stomach: A review of epidemiology, pathogenesis, molecular genetics and chemoprevention. World J Gastrointest Oncol, 4, 156-69.

Orazova G, Karp L, Yoo KY, et al (2015). Stomach cancer morbidity in the Republic of Kazakhstan: Trends and characteristics. Eur J Gen Med, 12, 282-90.

Quach DT, Hiyama T, Gotoda T (2019). Identifying high-risk individuals for gastric cancer surveillance from western and eastern perspectives: Lessons to learn and possibility to develop an integrated approach for daily practice. World $J$ Gastroenterol, 25, 3546-62.

Rawla P, Barsouk A (2019). Epidemiology of gastric cancer: global trends, risk factors and prevention. Prz Gastroenterol, 14, 26-38.

Sitarz R, Skierucha M, Mielko J, et al (2018). Gastric cancer: epidemiology, prevention, classification, and treatment. Cancer Manag Res, 10, 239-48.

Umarova G, Mamyrbayev A, Bermagambetova S, et al (2016). Morbidity, disability and death rates of the population due to malignant neoplasms in Uralsk City in The Republic of Kazakhstan. Asian Pac J Cancer Prev, 17, 5159-64.

van der Post RS, Vogelaar IP, Carneiro F, et al (2015). Hereditary diffuse gastric cancer: updated clinical guidelines with an emphasis on germline CDH1 mutation carriers. J Med Genet, 52, 361-74.

Vogelaar IP, van der Post RS, Bisseling TM, et al (2012). Familial gastric cancer: detection of a hereditary cause helps to understand its etiology. Hered Cancer Clin Pract, 10, 18.

Wang J, Sun Y, Bertagnolli MM (2015). Comparison of gastric cancer survival between Caucasian and Asian patients treated in the United States: results from the Surveillance Epidemiology and End Results (SEER) database. Ann Surg Oncol, 22, 2965-71.

Yang D, Hendifar A, Lenz C, et al (2011). Survival of metastatic gastric cancer: Significance of age, sex and race/ ethnicity. J Gastrointest Oncol, 2, 77-84.

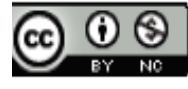

This work is licensed under a Creative Commons AttributionNon Commercial 4.0 International License. 